\title{
Posttranslational Protein Modification in the Salivary Glands of Sjögren's Syndrome Patients
}

\author{
Rafael Herrera-Esparza, Mayra Rodríguez-Rodríguez, María Elena Pérez-Pérez, \\ Martha Adriana Badillo-Soto, Felipe Torres-del-Muro, Juan José Bollain-y-Goytia, \\ Deyanira Pacheco-Tovar, and Esperanza Avalos-Díaz
}

\begin{abstract}
Department of Immunology, School of Biological Sciences, Universidad Autónoma de Zacatecas, Chepinque 306, Colonia Lomas de la Soledad, 98040 Zacatecas, ZAC, Mexico
\end{abstract}

Correspondence should be addressed to Rafael Herrera-Esparza; rafael.herreraesparza@gmail.com

Received 5 November 2012; Accepted 2 January 2013

Academic Editor: Ricard Cervera

Copyright (C) 2013 Rafael Herrera-Esparza et al. This is an open access article distributed under the Creative Commons Attribution License, which permits unrestricted use, distribution, and reproduction in any medium, provided the original work is properly cited.

\begin{abstract}
The present study investigated posttranslational reactions in the salivary glands of patients with Sjögren's syndrome. We analysed the biopsies of primary Sjögren's patients using immunohistochemistry and a tag-purified anticyclic citrullinated protein (CCP) antibody to detect citrullinated peptides, and the presence of peptidylarginine deiminase 2 (PAD2) was assessed simultaneously. The present work demonstrated the weak presence of the PAD2 enzyme in some normal salivary glands, although PAD2 expression was increased considerably in Sjögren's patients. The presence of citrullinated proteins was also detected in the salivary tissues of Sjögren's patients, which strongly supports the in situ posttranslational modification of proteins in this setting. Furthermore, the mutual expression of CCP and PAD2 suggests that this posttranslational modification is enzyme dependent. In conclusion, patients with Sjögren's syndrome expressed the catalytic machinery to produce posttranslational reactions that may result in autoantigen triggering.
\end{abstract}

\section{Introduction}

Sjögren's syndrome is an autoimmune epithelitis that primarily affects the salivary and lachrymal glands and results clinically in Sicca syndrome, which is characterised by xerostomia, xerophthalmia, and keratoconjunctivitis [1].

Citrulline is an $\alpha$-amino acid that can be isolated from watermelon [2]. In addition, it is an intermediate compound of the urea cycle and is synthesised in the liver by the conversion of ornithine to arginine during urea formation [3]. Citrulline exists in two forms: free citrulline, which is a product of the NOS enzyme family, and citrulline that results from the posttranslational modification of certain proteins at arginine residues, which is catalysed by peptidylarginine deiminases (PADs). This family of enzymes deiminates proteins at arginine residues to yield citrulline residues, and in this manner, protein citrullination is NOS independent [4].

We previously demonstrated inflammatory citrullination in the salivary glands of Sjögren's syndrome patients that was partially dependent on iNOS. This enzyme is overexpressed in inflammatory cells and infiltrates of the acini and salivary epithelia, and the in situ NO overdrive may contribute to glandular damage [5]. TNF and other proinflammatory cytokines are frequently observed in the glandular tissues of these patients, and these factors may trigger the production of iNOS [6]. Although protein citrullination is an important event in rheumatoid arthritis pathogenesis $[7,8]$, this process may also occur in Sjögren's syndrome. Approximately $7.5 \%$ of primary Sjögren's syndrome patients are positive for anticyclic citrullinated protein (anti-CCP) antibodies [9], and the presence of this marker in Sjögren's syndrome may be associated with nonerosive arthritis [10]. Protein citrullination has been shown to be an important pathophysiological mechanism in rheumatoid arthritis, and this process has also been reported in other autoimmune diseases such as antibody-associated neurodegenerative diseases [11]. Therefore, we investigated protein citrullination at the glandular level in Sjögren’s syndrome. 

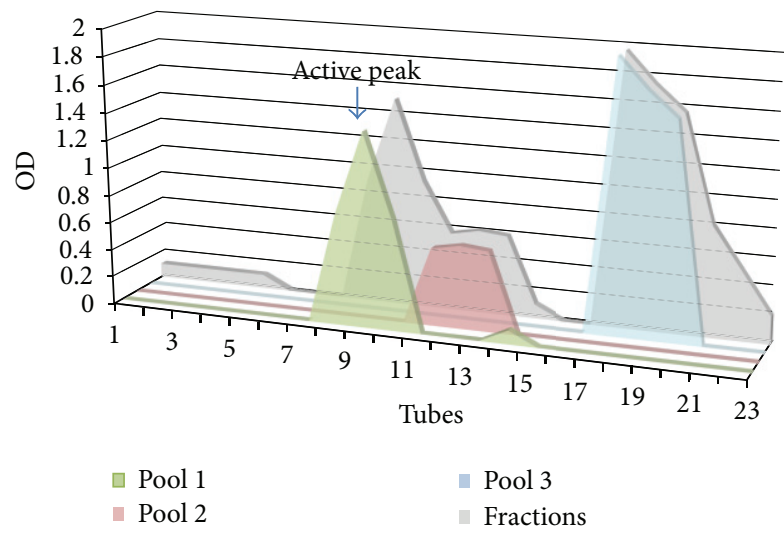

(a)

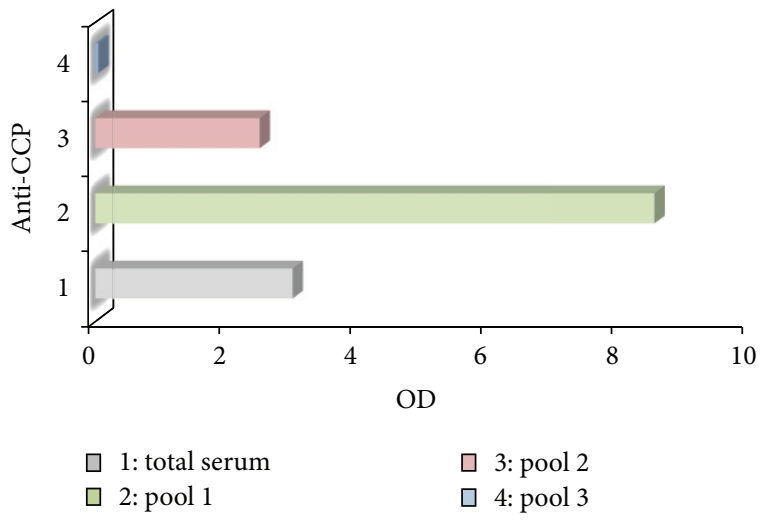

(b)

FIgure 1: (a) Chromatogram shows the purification of anti-CCP conjugated antibody that was further fractionated using gel filtration chromatography, which produced three fractions. The first pool (tubes 9-11) contained the HRP-anti-CCP conjugates (green) second pool (tubes 12-14) (pink). Pool 3 (tubes 18-20) contained the nonconjugated peroxidase fraction (blue). (b) ELISA graph of different fractions obtained by gel filtration. Pool 1 was very reactive for CCP, with an optical density that was threefold higher than the original serum titre. Pool 2 activity was weaker, and pool 3 was negative. (OD: optical density).

\section{Material and Methods}

2.1. Tissues. Minor salivary gland biopsies from 24 patients with primary Sjögren's syndrome were studied. The patients fulfilled the revised version of the European criteria proposed by the American-European Consensus [12]. This study included 17 women and seven men with a mean age of 47 years (range, 37-57 years). All of the patients and controls were informed of the biopsy, and written authorisation was obtained. An equal number of biopsies were obtained during oral surgery for other purposes from individuals without Sjögren's syndrome, who were included as controls. The ethics committee of our institution authorised and monitored the protocol, which conformed to the requirements of the World Medical Association's Declaration of Helsinki. The specimens were fixed in formaldehyde, embedded in paraffin, sectioned at a $4 \mu \mathrm{m}$ thickness, and stained with haematoxylin and eosin.
Two pathologists microscopically evaluated the biopsies, which were classified according to the revised multilevel assessment of a cumulative focus score [13]. Unstained tissue sections were used for immunohistochemistry.

2.2. Anti-CCP Antibodies. Enzyme immunoassays detected antibodies against citrullinated synthetic peptides from vimentin and filaggrin in sera from patients and controls. Serum from patients with rheumatoid arthritis was used as a positive control. The assays were performed according to the manufacturer's instructions (Euroimmun AG, Luebeck Germany). First, the serum samples were diluted 1:100 in dilution buffer. Second, the sera dilutions were incubated for 60 minutes at room temperature in wells of polystyrene plates coated with synthetic cyclic citrullinated peptides (CCPs), which contained arginine-modified residues. The citrullinated synthetic peptides included filaggrin, EBV nuclear antigen, and IgG sequences, and the CCPs included recombinant rat filaggrin or mutated human vimentin [14]. Third, the plates were washed with washing buffer. Fourth, the bound antibodies were tagged with the secondary antibody (conjugated POD-rabbit polyclonal anti-human IgG) during a 30minute incubation. Fifth, the colour reaction was developed during a 30-minute incubation with the colour developer pNPP ( $p$-nitrophenyl phosphate, disodium salt). Sixth, the reaction was stopped, and the microplates were evaluated using an ELISA reader at $450 \mathrm{~nm}$. Lastly, five calibrators were used to construct the curve analysis, and the results are expressed in units. All assays were performed in triplicate.

2.3. Purification of Affinity-Bound CCP Peptides. A rheumatoid arthritis patient who was positive for anti-CCP antibodies (filaggrin or mutated human vimentin) was submitted to ELISA, as described previously [15]. Specific anti-CCP bound antibodies were eluted from the polystyrene CCPcoated plates after a 2-hour incubation using $0.2 \mathrm{M}$ glycine$\mathrm{HCl}, \mathrm{pH} 2.8$, and the eluted antibodies were neutralised with $1 \mathrm{M}$ Tris, $\mathrm{pH}$ 9.5. The recovered antibodies were concentrated in a Centricon centrifugal device with a $30 \mathrm{kDa}$ molecular weight cut-off (Millipore).

2.4. Antibodies Labelling with Peroxidase. Protein concentrations of affinity-purified antibodies were measured using the Bradford method [16], and the high-affinity-purified antiCCP antibodies were labelled with horseradish peroxidase (HRP) (Sigma, St. Louis, MO) using the method described by Avrameas [17] with modifications. Briefly, the molar ratio of high-affinity-purified antibodies to peroxidase was 1:10. The enzyme was preactivated with glutaraldehyde and incubated at $37^{\circ} \mathrm{C}$ for 30 minutes. The coupling reaction was induced during an 18-hour incubation at room temperature in a sodium carbonate buffer, $\mathrm{pH}$ 9.5. The Anti-CCP/HRP was extensively dialysed against distilled $\mathrm{H}_{2} \mathrm{O}$ using a membrane with a pore size of $40 \mathrm{kDa}$. The anti-CCP/HRP conjugate was fractionated in a minicolumn packed with Sephadex G200 , which had been equilibrated with PBS, and an elution volume of $120 \mu \mathrm{L}$ per/fraction was collected in 25 tubes. The protein concentration was recorded in the different fractions, 

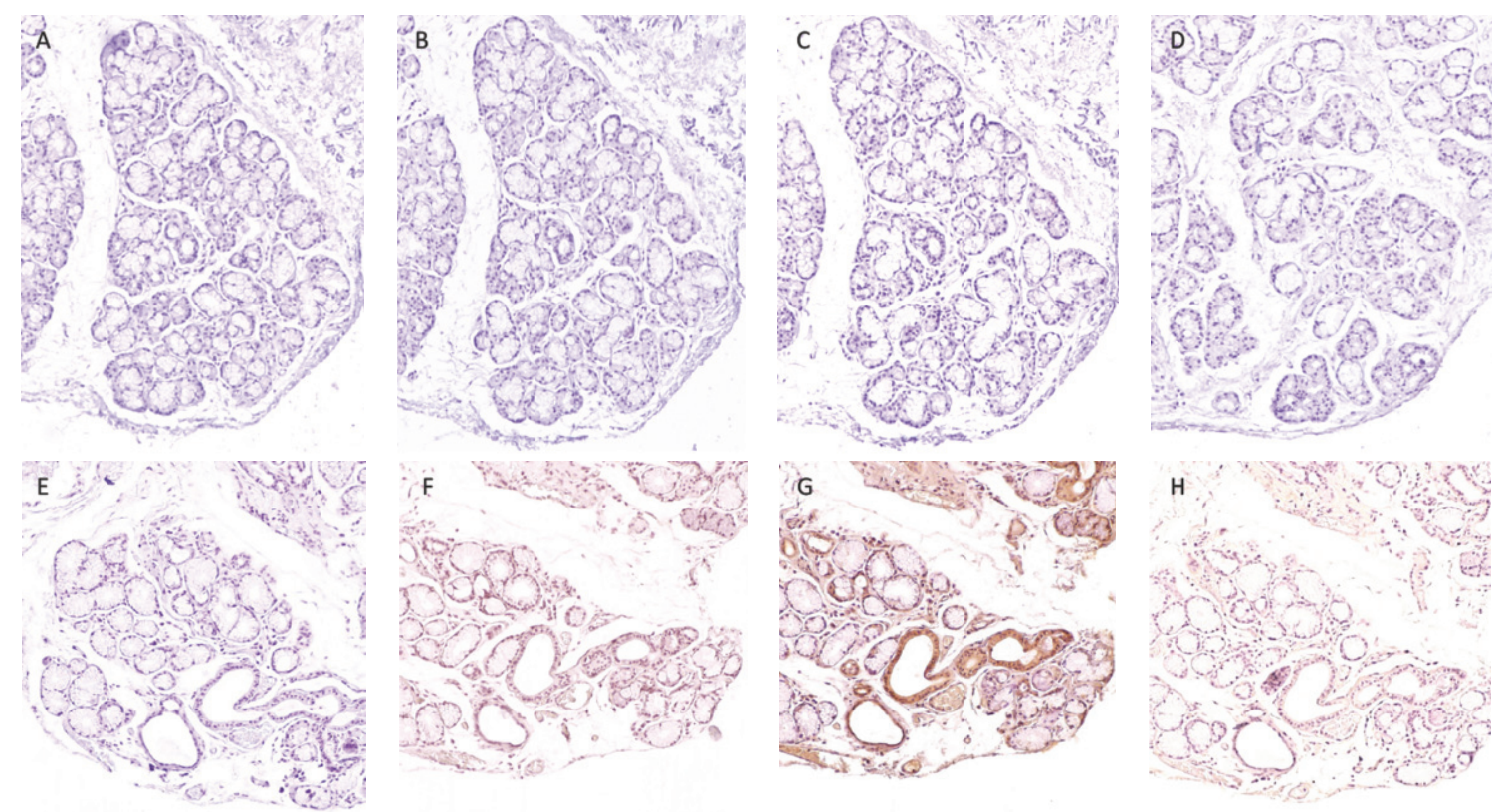

FIGURE 2: Immunohistochemistry of minor salivary glands. The superior panel corresponds to normal controls, and the inferior panel belongs to one representative biopsy of a Sjögren patient. (A) and (E). Incubated with PBS. (B) and (F). Treated with anti-CCP. (C) and (G). Anti-PAD2. (D) and (H). Anticitrulline. Observe the immunoreagents positivity at ducts and acini in (F), $(\mathrm{G})$, and $(\mathrm{H})$.

and three pools were created according to the chromatogram. Direct ELISA assays determined the reactivity to CCP of each pool, and each pool was concentrated to obtain $33 \%$ of the final volume using a Centricon device. The affinity anti-CCPpurified antibodies were used to trace CCP antigens in the salivary glands using immunohistochemistry.

2.5. Immunohistochemistry. Immunohistochemistry was performed to detect posttranslationally modified proteins in $4 \mu \mathrm{m}$-thick sections of fixed minor salivary glands on microscope slides. The specimens were deparaffinised, permeabilised with $0.01 \%$ Triton X-100/phosphate-buffered saline (PBS), and washed three times with PBS. Endogenous peroxidase was quenched for 10 minutes using $3 \% \mathrm{H}_{2} \mathrm{O}_{2}$ in methanol. The tissues were incubated with purified human IgG precipitated from normal human sera with ammonium sulphate to neutralise the presence of possible rheumatoid factor activity in salivary glands, and the precipitates were dialysed against distilled water using Slide-A-Lyzer Dialysis Cassettes (10 K MWCO Thermo Fisher Scientific Inc., Rockford, IL). IgG was purified using HiTrap protein G HP columns. The tissues were washed and incubated for 12 hours with an affinity-purified anti-CCP antibody (diluted 1:50) in $10 \%$ BFS-PBS. Bound anti-CCP antibodies were developed using 3,3'-diaminobenzidine- $0.06 \% \mathrm{H}_{2} \mathrm{O}_{2}$ (Sigma, St. Louis, MO) after washing, and the reaction was stopped with $0.5 \mathrm{M}$ sulphuric acid. The slides were examined under a light microscope. All assays were performed in triplicate and evaluated in a blinded manner. In addition, an anti-citrulline antibody (cat. 231246, Calbiochem, Darmstadt, Germany; $1: 100$ dilution in $10 \%$ foetal bovine serum (FBS)-PBS) and an anti-PAD 2 antibody (PA5-19474, Pierce; diluted 1:30) were tested on salivary glands in immunohistochemistry assays. These antibodies were incubated overnight, washed with PBS, and incubated with the secondary antibody (goat anti-rabbit IgG labelled with peroxidase; Abcam ab759). The colour reaction was induced as previously described and evaluated in a blinded manner [5].

2.6. Statistical Analyses. Data were processed using a Fisher's exact test using the GraphPad Software, QuickCalcs; the twotailed $P$ value $<0.005$ was considered statistically significant.

\section{Results}

3.1. Lymphocyte Infiltrates. The absence of any inflammatory infiltrate was classified as a score of 0 , which was characteristic of all control biopsies. Five biopsies from Sjögren's syndrome patients were scored as class I, and these biopsies exhibited discrete sialadenitis. Six biopsies were scored as class II and exhibited a moderate infiltration of mononuclear cells grouped in a single focus. Five biopsies were scored as class III and exhibited severe sialadenitis and one infiltrating focus. Eight biopsies were scored as class IV and displayed chronic sialadenitis with more than one foci (Table 1).

\subsection{Purification and Labelling of High-Affinity Anti-CCP} Antibodies. We purified anti-CCP antibodies, which were bound to commercial polystyrene plates covered with citrullinated synthetic peptides, from the total protein in each microwell. We recovered $30 \mu \mathrm{g} / \mathrm{well}$ of specific and highaffinity anti-CCP antibodies after glycine elution. We used the traditional coupling method of horseradish peroxidase bound to anti-CCP antibodies and glutaraldehyde at a molar 
TABle 1: Clinical, serologic, and immunohistochemistry data.

\begin{tabular}{|c|c|c|c|c|c|c|c|}
\hline \multicolumn{4}{|c|}{ Sjögren $(n=24)$} & \multicolumn{4}{|c|}{ Controls $(n=24)$} \\
\hline \multicolumn{8}{|l|}{ Clinical data } \\
\hline Sicca & \multicolumn{3}{|c|}{$24(8=\mathrm{IV}, 5=\mathrm{III}, 6=\mathrm{II}, 5=\mathrm{I})$} & \multicolumn{3}{|c|}{0} & ${ }^{*} P<0.0001$ \\
\hline Glandular & \multicolumn{3}{|c|}{$24(8=\mathrm{IV}, 5=\mathrm{III}, 6=\mathrm{II}, 5=\mathrm{I})$} & \multicolumn{3}{|c|}{0} & ${ }^{*} P<0.0001$ \\
\hline Extraglandular & \multicolumn{3}{|c|}{$10(8=\mathrm{IV}, 2=\mathrm{III})$} & \multicolumn{3}{|c|}{0} & ${ }^{*} P<0.0006$ \\
\hline \multicolumn{8}{|l|}{ Serology } \\
\hline Anti-CCP & \multicolumn{3}{|c|}{$2(2=\mathrm{IV})$} & \multicolumn{3}{|c|}{0} & ${ }^{*} P<0.4890$ \\
\hline$+\mathrm{RF}$ & \multicolumn{3}{|c|}{$10(8=\mathrm{IV}, 2=\mathrm{III})$} & \multicolumn{3}{|c|}{0} & ${ }^{*} P<0.0006$ \\
\hline+ ANA & \multicolumn{3}{|c|}{$16(8=\mathrm{IV}, 5=\mathrm{III}, 3=\mathrm{II})$} & \multicolumn{3}{|c|}{0} & ${ }^{*} P<0.0001$ \\
\hline+ Anti-La & \multicolumn{3}{|c|}{$12(7=\mathrm{IV}, 4=\mathrm{III}, 1=\mathrm{II})$} & \multicolumn{3}{|c|}{0} & ${ }^{*} P<0.0025$ \\
\hline+ Anti-Ro & \multicolumn{3}{|c|}{$14(8=\mathrm{IV}, 4=\mathrm{III}, 1=\mathrm{II}, 1=\mathrm{I})$} & \multicolumn{3}{|c|}{0} & ${ }^{*} P<0.0001$ \\
\hline \multicolumn{8}{|l|}{ Biopsies } \\
\hline & Acini & Ducts & Infiltrates & Acini & Ducts & Infiltrates & \\
\hline PAD 2 & $\begin{array}{c}17 \\
{ }^{E}(8=\mathrm{IV}, 6=\mathrm{III}, 2=\mathrm{II}, 1=\mathrm{I})\end{array}$ & $\begin{array}{c}17 \\
{ }^{E}(8=\mathrm{IV}, 6=\mathrm{III}, 2=\mathrm{II}, 1=\mathrm{I})\end{array}$ & $\begin{array}{c}12 \\
\left.\mathcal{E}_{(8=\mathrm{IV}, 4}, 4=\mathrm{III}\right)\end{array}$ & 0 & 2 & 0 & ${ }^{*} P<0.0001$ \\
\hline $\mathrm{CCP}$ & ${ }^{E}(8=\mathrm{IV}, 5=\mathrm{III}, 3=\mathrm{II}, 1=\mathrm{I})$ & ${ }^{E}(8=\mathrm{IV}, 5=\mathrm{III}, 3=\mathrm{II}, 1=\mathrm{I})$ & ${ }^{\mathrm{E}}(8=\mathrm{IV}, 5=\mathrm{III}, 3=\mathrm{II}, 1=\mathrm{I})$ & 0 & 0 & 0 & ${ }^{*} P<0.0001$ \\
\hline Citrulline & $\begin{array}{c}19 \\
{ }^{E}(8=\mathrm{IV}, 5=\mathrm{III}, 5=\mathrm{II}, 1=\mathrm{I})\end{array}$ & $\stackrel{19}{{ }^{\mathfrak{E}}(8=\mathrm{IV}, 5=\mathrm{III}, 5=\mathrm{II}, 1=\mathrm{I})}$ & 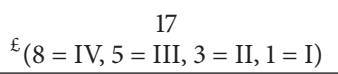 & 0 & 2 & 0 & ${ }^{*} P<0.0001$ \\
\hline
\end{tabular}

${ }^{*}$ Sjögren versus controls.

${ }^{E}$ Sjögren class IV versus class I $=P<0.003$.

Histology class inside the parentheses.

ratio of enzyme: antibody of $10: 1$, which worked well. The conjugated antibody was further fractionated using gel filtration chromatography, which produced three fractions. The first fraction pool contained the HRP-anti-CCP conjugates (fractions 9-11 or pool 1), and this fraction was very reactive to CCP by ELISA, with an optical density that was threefold higher than the original serum titre. The anti-CCP activity was weaker in the second pool (fractions 12-14 or pool 2). Pool 3 (fractions 18-20) contained the nonconjugated peroxidase fraction, which was negative for anti-CCP by ELISA (Figure 1).

3.3. Anti-CCP Antibodies. Two of the samples from primary Sjögren's syndrome patients were positive for anti-CCP antibodies in the serum, whereas healthy controls were negative for anti-CCP antibodies.

3.4. Normal Salivary Glands Express PAD2. The peptidylarginine deiminases belong to a family of enzymes that posttranslationally modify proteins at positively charged arginine residues to neutral citrulline residues via a citrullination process. These enzymes play a role in various physiological processes, and they are widely distributed in tissues. Therefore, we investigated the distribution of these enzymes in normal salivary glands and observed the discrete presence of PAD2 in the ductal epithelia of selected minor salivary gland of some biopsies. However, expression of this enzyme was absent in the acini.

3.5. Protein Posttranslational Modification Occurs in the Salivary Glands of Sjögren's Syndrome Patients. We next examined the presence of posttranslationally citrullinated proteins in the salivary glands of primary Sjögren's syndrome patients using our high-affinity-purified anti-CCP antibodies. We detected the presence of posttranslationally modified proteins in the salivary ducts, acini, and inflammatory infiltrate foci of $70 \%$ of the Sjögren's syndrome patient biopsies, although evidence for this citrullination process was absent in normal control samples, $P$ value $<0.0001$ (Figures 2 and 3 and Table 1).

3.6. Protein Citrullination Is PAD2 Dependent. The distribution of PAD2 was then compared to the distribution of citrullinated peptides (according to anti-CCP labelling) to determine the association between this enzyme and in situ citrullination. We observed total agreement between the presence of both factors, which suggested that this expression was consistent with the extensive areas of citrullination in the ducts, acini, and inflammatory infiltration foci. These results strongly support the in situ posttranslational modification of proteins (Figure 4).

\section{Discussion}

The antigenic triggering by posttranslational modifications of normal proteins is an important component of autoimmune diseases. This process transforms certain protein epitopes that may trigger an autoimmune response in genetically susceptible individuals. The present study investigated whether the salivary glands in Sjögren's syndrome patients are potential sources of posttranslational protein modification. Biopsies from primary Sjögren's syndrome patients were used to detect posttranslationally modified proteins using a taginduced anti-CCP antibody and immunohistochemistry. The potential role of the PAD2 enzyme as the catalyst for these modifications was also assessed. The primary results of the present study can be summarised as follows. First, the PAD2 enzyme was detected in some normal salivary glands, 


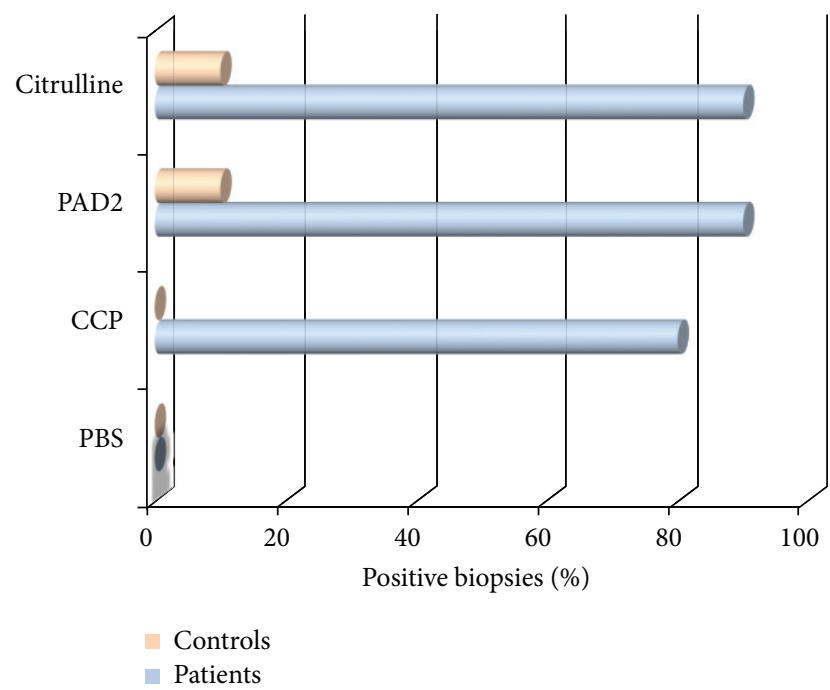

(a)

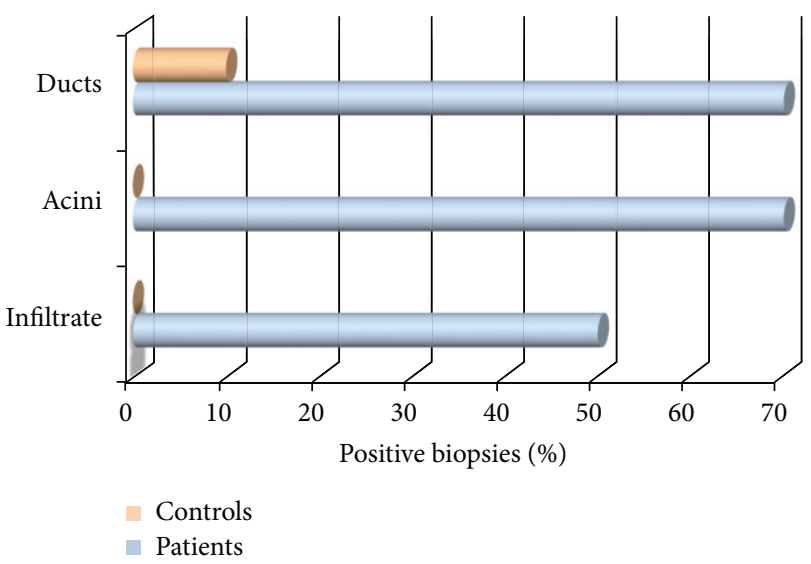

(b)

FIGURE 3: (a) Comparative immunohistochemistry assay of CCP, PAD2, and citrulline in controls and Sjögren patients; note that $10 \%$ of normal biopsies express the PAD2 enzyme, while Sjögren patients exclusively express CCP mutually distributed with PAD2. (b) Distribution of CCP/PAD2 in compartments of salivary glands of Sjögren patients.

but its expression was increased considerably in Sjögren's syndrome patients. Second, the presence of citrullinated proteins in the salivary tissues of Sjögren's patients strongly supports the posttranslational modification of proteins in situ. Third, the mutual expression of CCP and PAD2 suggests that posttranslational modifications are enzyme dependent. Finally, the inflammatory infiltrates were associated with PAD2 overexpression, which was directly related to the presence of protein modifications.

The PAD2 enzyme isoform is extensively distributed in a variety of mammalian tissues, including the nervous system, skeletal muscle, pancreas, sweet glands, salivary glands, mammary fat pads, bone marrow, and immune cells such as monocytes and macrophages [18-20]. The salivary glands broadly express proteins that may be modified by
PAD, including vimentin, enolase, and others [21-23]. Furthermore, the salivary glands constitutively express PAD2, and this enzyme may convert arginine residues into citrulline, which is the cornerstone of the citrullination process. Additionally, the epithelial and glandular components of salivary glands express vimentin, enolase, and other proteins that are potential substrates for PAD2, and some of these proteins have been recognised as autoantigens in autoimmune diseases, including rheumatoid arthritis. Therefore, we assumed that all of the necessary elements for posttranslational modification existed, and these molecules were observed to be present in the salivary glands of patients with Sjögren's syndrome. Following this posttranslational process, modified proteins have been shown to trigger autoimmune responses. The first evidence for antibody generation in response to modified cellular components was reported as the presence of autoantibodies against filaggrin, which is detected in normal epithelial cells of the oral mucosa. Therefore, this autoantibody was termed as an "antiperinuclear factor" and was shown to be present in rheumatoid arthritis patients. Similarly, anti-CCP antibodies serve as an early marker of rheumatoid arthritis, and the importance of anti-CCP antibodies in disease pathogenesis has been increasingly demonstrated [24-28]. Furthermore, posttranslational protein modifications may trigger other autoimmune diseases, such as optic neuritis, coeliac disease, and multiple sclerosis [29-31].

The biggest challenge in the current study was the poor availability of anti-CCP reagents from a commercial source. Therefore, we obtained autoantibodies from rheumatoid arthritis patients to perform our studies. We were inspired by the study of Vossenaar et al. [32], as these authors obtained affinity-purified antibodies from the serum of rheumatoid arthritis patients using an immunoadsorption technique with recombinant peptides that were produced by phage display technology. We used a very simple technology that exploited the ability of immobilised synthetic peptides on polystyrene commercial plates to bind high-affinity anti-CCP antibodies, which were eluted from plates with a glycine buffer that lowers the $\mathrm{pH}$. This method recovered a useful amount of high-affinity anti-CCP antibodies, which demonstrated a threefold increased reactivity to citrullinated synthetic peptides in commercial ELISA plates. These high-affinity anti-CCP antibodies were conjugated to peroxidase, which served as an excellent tag for CCP, as demonstrated in the immunohistochemistry assays; efficiency of our antibodies is comparable to that previously reported by other authors [32]. Furthermore, potential false positives for the presence of rheumatoid factor were prevented by the preincubation with normal IgG, which neutralised the rheumatoid factor activity.

Our results are important for understanding Sjögren's syndrome pathophysiology because these modifications constitute an additional mechanism for the triggering of autoimmunity. These modifications may also be applicable to other autoimmune diseases because posttranslational modification may represent a common mechanism for the genesis of autoantigens. For example, the study by Routsias et al. demonstrated that the posttranslational modification of the auto-antigen La increased its antigenicity $[33,34]$. These 

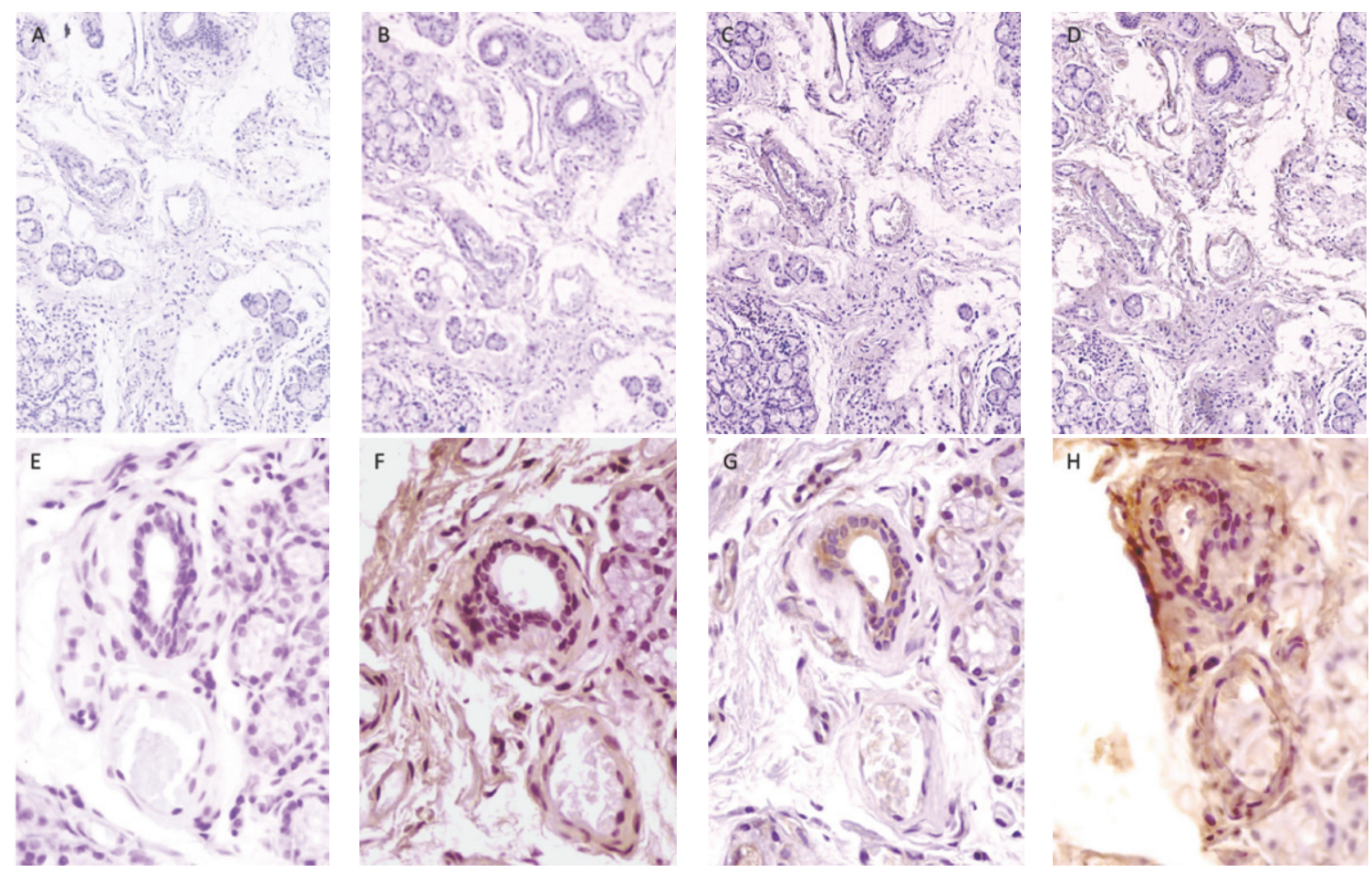

FIGURE 4: Immunohistochemistry of minor salivary glands from Sjögren patients; the superior panel shows an overview of immunoreagent distribution at 10x magnification; the inferior panel showed a close up at 40x magnification. (A) and (E). Incubated with PBS. (B) and (F). Treated with anti-CCP. (C) and (G). Anti-PAD2. (D) and (H). Anticitrulline. Note the correlation between CCP, PAD2, and citrulline expression in a duct.

results support a new perspective for exploring the origins of autoimmunity based on antigenic triggering in genetically susceptible individuals.

However, many questions remain unresolved. For example, do the constitutively expressed PAD enzymes in normal glandular epithelia (PAD2) play a role in the initial triggering of autoimmunity in Sjögren's syndrome? Additionally, do previous triggers induce inflammatory foci formation and PAD2 overexpression in mononuclear cells and macrophages leading to the posttranslational modification of salivary gland proteins? Both of these possibilities may coexist and create a feedback loop, although the theoretical aspects of this hypothesis require clarification using other experimental approaches.

\section{Conclusions}

In conclusion, Sjögren's syndrome patients possess the catalytic machinery to posttranslationally modify proteins, which may result in auto-antigen triggering.

\section{Acknowledgments}

This work was supported by PROMEP-UAZ-CA-5 Autoinmunidad, Grant no. 103.5/09/1327, and Red temática: Autoinmunidad multicéntrica. The authors thank Lizeth Ivon Alvarez-Cháirez and Sara Paola Hernández-Martínez their valuable help in some experiments.

\section{References}

[1] H. M. Moutsopoulos, “Sjogren's syndrome: autoimmune epithelitis," Clinical Immunology and Immunopathology, vol. 72, no. 2, pp. 162-165, 1994.

[2] M. Wada, "Über citrullin, eine neue aminosäure im presssaft der wassermelone, citrullus vulgaris schrad," Biochemische Zeitschrift, vol. 224, pp. 420-429, 1930.

[3] H. A. Krebs and K. Henseleit, "Studies on urea formation in the animal organism," Hoppe-Seyler's Zeitschrift fur Physiologische Chemie, vol. 210, pp. 33-66, 1932.

[4] E. Curis, I. Nicolis, C. Moinard et al., "Almost all about citrulline in mammals," Amino Acids, vol. 29, no. 3, pp. 177-205, 2005.

[5] J. J. Bollain-Y-Goytia, L. Mendoza-Salazar, M. D. L. RomoFlores et al., "The presence of citrulline in salivary glands is evidence that nitric oxide is mediator of inflammation in Sjögren acinar epithelia," International Journal of Rheumatic Diseases, vol. 11, no. 3, pp. 263-268, 2008.

[6] I. Badillo-Almaraz, E. Avalos-Díaz, R. Villalobos-Hurtado, and R. Herrera-Esparza, "FasL and Bax genes are differentially expressed in acinar epithelium and inflammatory cells of primary Sjögren glands," Minerva Medica, vol. 94, no. 5, pp. 341345, 2003.

[7] J. J. B. C. van Beers, R. Raijmakers, L. E. Alexander et al., "Mapping of citrullinated fibrinogen B-cell epitopes in rheumatoid arthritis by imaging surface plasmon resonance," Arthritis Research \& Therapy, vol. 12, no. 6, article R219, 2010.

[8] J. J. van Beers, A. Willemze, J. Stammen-Vogelzangs, J. W. Drijfhout, R. E. Toes, and G. J. Pruijn, "Anti-citrullinated fibronectin antibodies in rheumatoid arthritis are associated 
with human leukocyte antigen-DRB1 shared epitope alleles," Arthritis Research \& Therapy, vol. 14, no. 1, article R35, pp. 1-16, 2012.

[9] J. E. Gottenberg, S. Mignot, P. Nicaise-Rolland et al., "Prevalence of anti-cyclic citrullinated peptide and anti-keratin antibodies in patients with primary Sjögren's syndrome," Annals of the Rheumatic Diseases, vol. 64, no. 1, pp. 114-117, 2005.

[10] F. Atzeni, P. Sarzi-Puttini, N. Lama et al., "Anti-cyclic citrullinated peptide antibodies in primary Sjögren syndrome may be associated with non-erosive synovitis," Arthritis Research \& Therapy, vol. 10, no. 3, article R51, 2008.

[11] N. K. Acharya, E. P. Nagele, M. Han et al., "Neuronal PAD4 expression and protein citrullination: possible role in production of autoantibodies associated with neurodegenerative disease," Journal of Autoimmunity, vol. 38, no. 4, pp. 369-380, 2012.

[12] C. Vitali, S. Bombardieri, R. Jonsson et al., "European Study Group on Classification Criteria for Sjögren's Syndrome. Classification criteria for Sjögren's syndrome: a revised version of the European criteria proposed by the American-European Consensus Group," Annals of the Rheumatic Diseases, vol. 61, no. 6, pp. 554-558, 2002.

[13] D. M. Chisholm and D. K. Mason, "Labial salivary gland biopsy in Sjögren's syndrome," Journal of Clinical Pathology, vol. 21, no. 5, pp. 656-660, 1968.

[14] N. Bizzaro, E. Tonutti, R. Tozzoli, and D. Villalta, "Analytical and diagnostic characteristics of 11 2nd- and 3rd-generation immunoenzymatic methods for the detection of antibodies to citrullinated proteins," Clinical Chemistry, vol. 53, no. 8, pp. 1527-1533, 2007.

[15] R. Herrera Esparza, T. Swaak, L. Aarden, and R. Smeenk, "Complement-fixing antibodies to dsDNA detected by the immunofluorescence technique on Crithidia luciliae. A critical appraisal," Journal of Rheumatology, vol. 12, no. 6, pp. 1109-1117, 1985.

[16] M. M. Bradford, "A rapid and sensitive method for the quantitation of microgram quantities of protein utilizing the principle of protein dye binding," Analytical Biochemistry, vol. 72, no. 1-2, pp. 248-254, 1976.

[17] S. Avrameas and T. Ternynck, "Peroxidase labelled antibody and Fab conjugates with enhanced intracellular penetration," Immunochemistry, vol. 8, no. 12, pp. 1175-1179, 1971.

[18] S. Horibata, S. A. Coonrod, and B. D. Cherrinton, "Role for the peptydilarginine deiminase in disease and female reproduction," The Journal of Reproduction and Development, vol. 58, no. 3, pp. 274-282, 2012.

[19] H. Terakawa, H. Takahara, and K. Sugawara, "Three types of mouse peptidylarginine deiminase: characterization and tissue distribution," Journal of Biochemistry, vol. 110, no. 4, pp. 661666, 1991.

[20] E. R. Vossenaar, T. R. D. Radstake, A. van der Heijden et al., "Expression and activity of citrullinating peptidylarginine deiminase enzymes in monocytes and macrophages," Annals of the Rheumatic Diseases, vol. 63, no. 4, pp. 373-381, 2004.

[21] T. Hjelmervik, R. Jonsson, and A. Bolstad, "The minor salivary gland proteome in Sjögren's syndrome,” Oral Diseases, vol. 15, no. 5, pp. 342-353, 2009.

[22] H. Dang, A. L. Lin, B. Zhang, H. M. Zhang, M. S. Katz, and C. K. Yeh, "Role for Notch signaling in salivary acinar cell growth and differentiation," Developmental Dynamics, vol. 238, no. 3, pp. 724-731, 2009.
[23] G. P. Harvey, T. R. Fitzsimmons, A. A. Dhamarpatni, C. Marchant, D. R. Haynes, and P. M. Bartold, "Expression of peptidylarginine deiminase- 2 and -4 , citrullinated proteins and anti-citrullinated protein antibodies in human gingiva," Journal of Periodontal Research, 2012.

[24] R. L. Nienhuis and E. Mandema, "A new serum factor in patients with rheumatoid arthritis; the antiperinuclear factor," Annals of the Rheumatic Diseases, vol. 23, pp. 302-305, 1964.

[25] R. M. A. Hoet, T. A. M. Boerbooms, M. Arends, D. J. Ruiter, and W. J. van Venrooij, "Antiperinuclear factor, a marker autoantibody for rheumatoid arthritis: colocalisation of the perinuclear factor and profilaggrin," Annals of the Rheumatic Diseases, vol. 50, no. 9, pp. 611-618, 1991.

[26] G. A. Schellekens, B. A. W. de Jong, F. H. J. van Den Hoogen, L. B. A. van De Putte, and W. J. van Venrooij, "Citrulline is an essential constituent of antigenic determinants recognized by rheumatoid arthritis-specific autoantibodies," Journal of Clinical Investigation, vol. 101, no. 1, pp. 273-281, 1998.

[27] N. Després, G. Talbot, B. Plouffe, G. Boire, and H. A. Ménard, "Detection and expression of a cDNA clone that encodes a polypeptide containing two inhibitory domains of human calpastatin and its recognition by rheumatoid arthritis sera," Journal of Clinical Investigation, vol. 95, no. 4, pp. 1891-1896, 1995.

[28] E. R. Vossenaar, N. Després, E. Lapointe et al., "Rheumatoid arthritis specific anti-Sa antibodies target citrullinated vimentin," Arthritis Research \& Therapy, vol. 6, no. 2, pp. R142R150, 2004.

[29] H. A. Doyle and M. J. Mamula, "Post-translational protein modifications in antigen recognition and autoimmunity," Trends in Immunology, vol. 22, no. 8, pp. 443-449, 2001.

[30] L. M. Sollid, "Coeliac disease: dissecting a complex inflammatory disorder," Nature Reviews Immunology, vol. 2, no. 9, pp. 647-655, 2002.

[31] M. T. Bedford and S. Richard, "Arginine methylation: an emerging regulator of protein function," Molecular Cell, vol. 18, no. 3, pp. 263-272, 2005.

[32] E. R. Vossenaar, T. J. M. Smeets, M. C. Kraan, J. M. Raats, W. J. van Venrooij, and P. P. Tak, "The presence of citrullinated proteins is not specific for rheumatoid synovial tissue," Arthritis and Rheumatism, vol. 50, no. 11, pp. 3485-3494, 2004.

[33] E. A. Stea, J. G. Routsias, M. Samiotaki et al., "Analysis of parotid glands of primary Sjögren's syndrome patients using proteomic technology reveals altered autoantigen composition and novel antigenic targets," Clinical and Experimental Immunology, vol. 147, no. 1, pp. 81-89, 2007.

[34] J. G. Routsias and A. G. Tzioufas, "Autoimmune response and target autoantigens in Sjogren's syndrome," European Journal of Clinical Investigation, vol. 40, no. 11, pp. 1026-1036, 2010. 


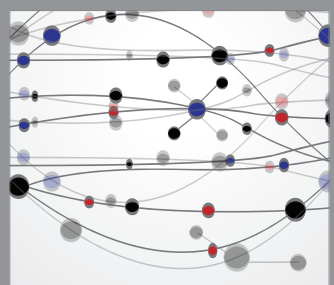

The Scientific World Journal
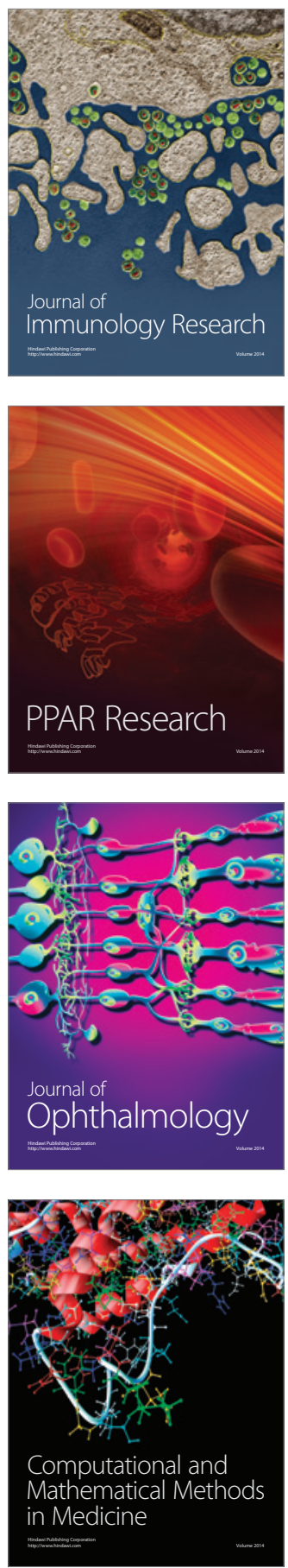

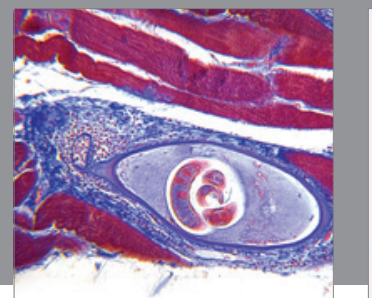

Gastroenterology

Research and Practice
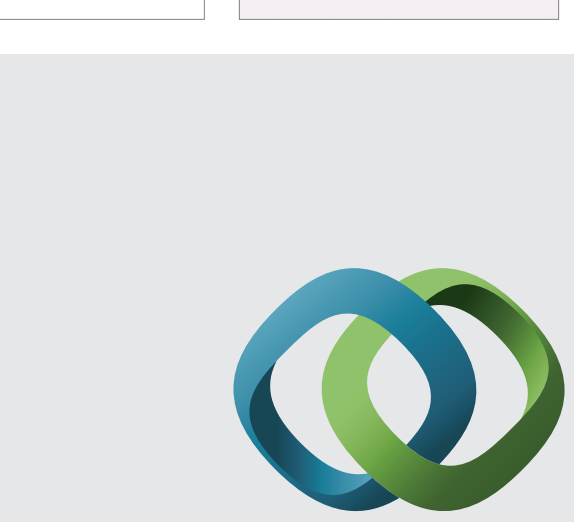

\section{Hindawi}

Submit your manuscripts at

http://www.hindawi.com
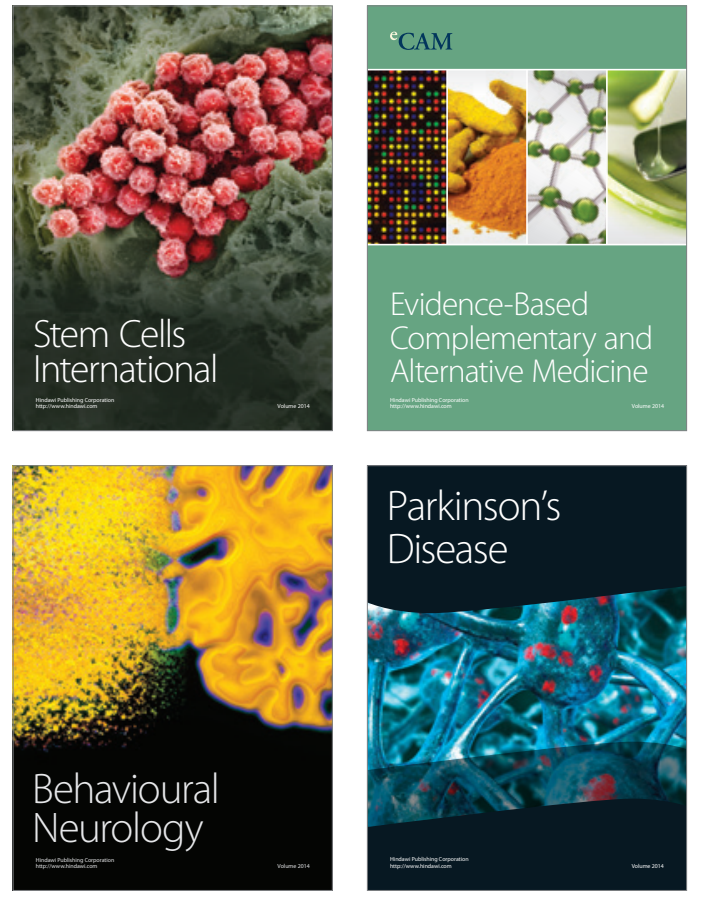
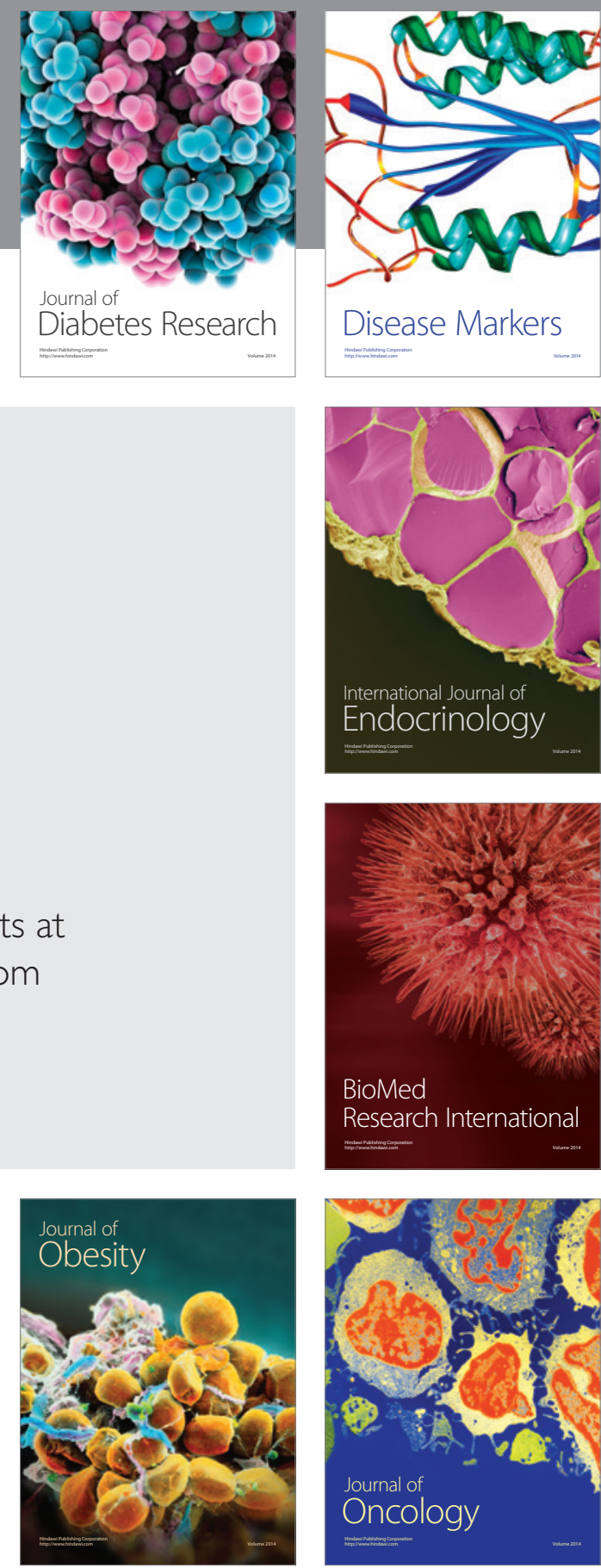

Disease Markers
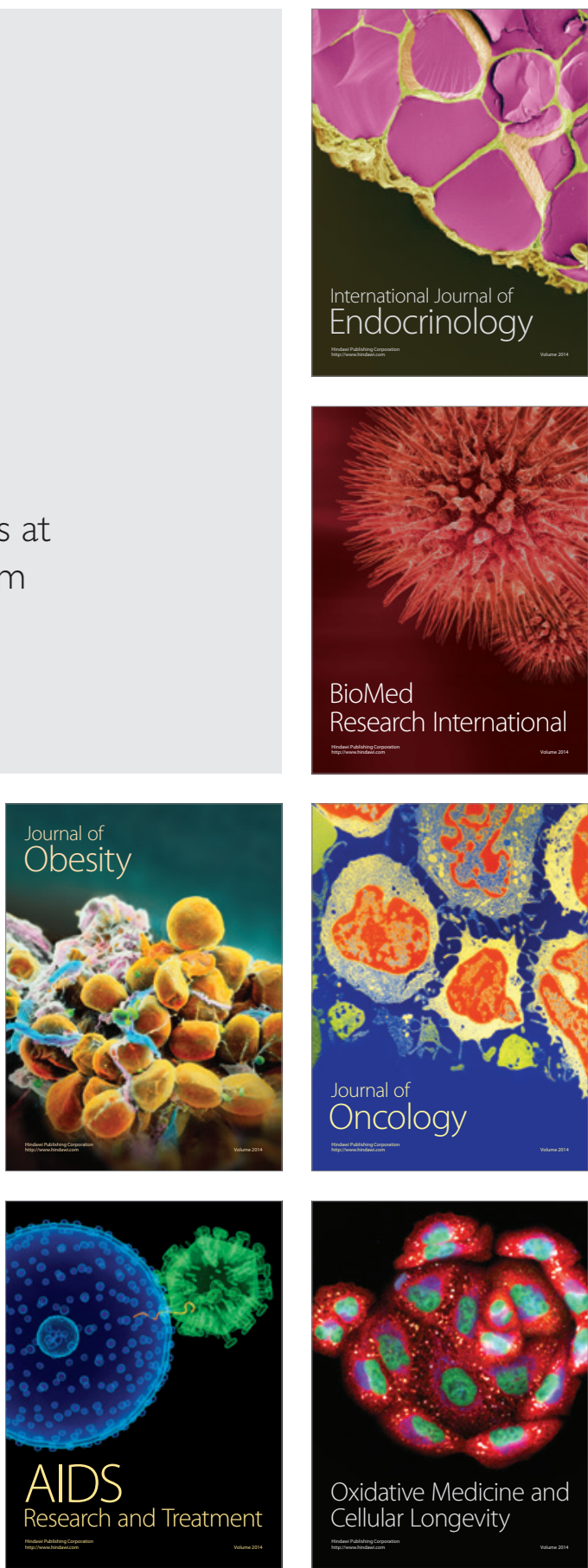